\title{
Fabrication and Properties of ac-dc Superconducting Rectifier
}

\author{
E. M. González, J. E. Villegas, M. P. González, J. V. Anguita, and J. L. Vicent
}

\begin{abstract}
Magnetron sputtering, electron beam lithography and etching techniques allow us to fabricate $\mathrm{Nb}$ thin films on array of periodic nonsuperconducting nanometric triangles. These asymmetric pinning potentials induce a ratchet effect on the vortex lattice motion: injecting an ac current on the sample yields a dc voltage. This rectification effect does not depend on the ac frequency in the $\mathrm{kHz}$ range. The effect occurs for magnetic as well as nonmagnetic pinning centers. The rectification is enhanced at lower temperatures.
\end{abstract}

Index Terms-Rectifiers, superconducting device fabrication, superconducting devices, superconducting films.

\section{INTRODUCTION}

$\mathbf{N}$ ANOTECHNOLOGY is one of the main subjects in today applied research. In the submicrometric scale, superconducting devices could be very useful tools in fields such as magnetoelectronic or spintronic. One of the most serious limits is related with the physical lengths that govern the superconducting properties. For example, the penetration depth and the coherence lengths are in the submicrometric range; hence the superconducting properties could be changed if the superconducting material is patterned into nanometric range sizes. In this work, we will show that introducing arrays of submicrometric ordered defects in a superconducting film could be a way to fabricate novel microdevices without deteriorating the superconducting properties of the film.

In a recent publication, Villegas et al. [1] have reported on the fabrication of a superconducting microdevice that allows to convert an ac injected current into a dc voltage. This superconducting device is based on the behavior of the vortex lattice moving on an array of periodic asymmetric pinning potentials. The injection of an ac current through the film produces a net drift of vortices, thus a dc voltage can be measured. This is a very general effect that is not restricted to superconductivity at all. In general, net dc motion is induced on particles, under nonequilibrium fluctuations, if they are moving on asymmetric potentials although no dc driving force is applied to the system. This is the well-known ratchet effect. This effect has

Manuscript received October 4, 2004. This work was supported by the Spanish Ministerio de Educación y Ciencia (MAT2002-04543, MAT200212385-E), the R. Areces Foundation, and CAM (GR/MAT/0617/2004). The work of E. M. González was supported by the Spanish Ministerio de Educación y Ciencia through a Ramón y Cajal contract.

E. M. González, J. E. Villegas, M. P. González and J. L. Vicent are with the Departamento Física Materiales, Universidad Complutense, Madrid 28040, Spain (e-mail: cygnus@fis.ucm.es; jeville@fis.ucm.es; mpgonzalez@ fis.ucm.es; jlvicent@ fis.ucm.es).

J. V. Anguita is with the Instituto Microelectrónica (CSIC), 28760 Madrid, Spain (e-mail: anguita@imm.cnm.csic.es).

Digital Object Identifier 10.1109/TASC.2005.850103 been studied in very different fields, from particles separation [1] to tunneling effects in semiconductor heterostructures [2]. In superconductors, several theoretical models have been published, most of them concerning the manipulation of vortices in nanometric patterned superconductors; for instance, to remove flux trapped in superconducting devices [3] or fluxon optic [4], [5]. From the experimental point of view the ratchet effect has been reported related to superconducting circuits [6]-[8], and very recently to superconducting devices based on low temperature superconductors [1], and high temperature superconductors [9]. The former is an ac-dc rectifier based on a $\mathrm{Nb}$ film grown on an array of Ni triangles. In this paper we will explore the experimental temperature and frequency windows and the role played by magnetic or nonmagnetic asymmetric potentials in this device. Therefore, we will address crucial parameters for the possible applications of this device.

\section{EXPERIMENT}

The samples are fabricated following several steps [10]. First, the pattern (in this case array of submicrometric triangles) is drawn with the electron beam of a scanning electron microscope onto a $\mathrm{Si}(100)$ substrate covered with an electron sensitive resist layer. After that, this resist layer is developed, so those regions which were exposed to the electron beam are removed from the substrate. Afterwards $\mathrm{Ni}$ or $\mathrm{Cu}$ are grown by magnetron sputtering on the substrate. Finally, a lift-off process is done and the deposited material only remains in those areas which are in contact with the substrate, i. e. the desired pattern (defined by the electron beam writing) is obtained. The usual size of the pattern is $90 \mu \mathrm{m} \times 90 \mu \mathrm{m}$. On top of this (array of triangles), a Nb film is deposited by magnetron sputtering. The thickness of the $\mathrm{Nb}$ film is always $100 \mathrm{~nm}$ and the thickness of the nanotriangles is always $40 \mathrm{~nm}$. The separation between triangles and the triangle side vary from sample to sample. In summary, we have fabricated superconducting $\mathrm{Nb}$ films on top of periodic arrays of pinning centers (nanotriangles of $\mathrm{Ni}$ or $\mathrm{Cu}$ ).

Transport measurements were carried out on $40 \mu \mathrm{m} \times 40 \mu \mathrm{m}$ cross-shaped bridge defined by conventional optical lithography and ion etching. This bridge allows us to inject the current either parallel or perpendicular to the triangular base. Magnetotransport experiments were done using a commercial liquid $\mathrm{He}$ cryostate with a superconducting solenoid. The magnetic field was applied perpendicular to the $\mathrm{Nb}$ film.

Vortex lattice motion in superconductors grown on arrays of periodic pinning potentials shows very interesting effects. For instance, dissipation minima (therefore critical current maxima) appear when the applied magnetic field induces a vortex lattice which matches the unit cell array [11]. In these conditions, 


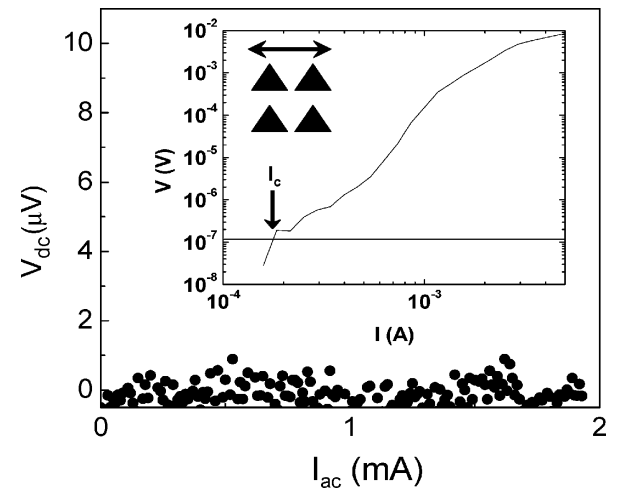

Fig. 1. Data obtained for a $\mathrm{Nb}$ film deposited on an array of Ni triangles $(620 \mathrm{~nm}$ side size) with periods of $770 \mathrm{~nm} \times 746 \mathrm{~nm}$. The critical temperature of the sample is $T_{c}=8.34 \mathrm{~K}$. The main graph shows the dc voltage versus ac current amplitude for an applied magnetic field $\mathrm{B}=32 \times 10^{-3} \mathrm{~T}(\mathbf{n}=1$, one vortex per unit cell). The ac current frequency is $\mathrm{f}=10 \mathrm{kHz}$ and the temperature is $T=0.99 \mathrm{~T}_{\mathrm{c}}$. Inset shows the $\mathrm{V}(\mathrm{I})$ curve and the position of the critical current $\mathrm{I}_{c}$ (marked with an arrow), the direction of vortex motion (double arrow) is indicated too.

vortex lattice motion is slow down and we can calculate both the number of vortices per unit cell and their positions inside the cell [12]. Hence, for selected values of the magnetic field, we are able to tune the total number of vortices per unit cell of the array and, at the same time, to distinguish between the number of trapped vortices in the pinning centers and the number of interstitial vortices.

\section{EXPERIMENTAL RESULTS AND DISCUSSION}

The dynamics of the vortex lattice on arrays of periodic asymmetric pinning centers could be a very useful tool to study the response of these particles to a ratchet-like potential. Following the process sketches in the previous section we have fabricated $\mathrm{Nb}$ thin film on arrays of nanometric Ni triangles. Magnetoresistance measurements allow us to know matching field values [1]. The number of vortices per array unit cell could be controlled and therefore it is easier to figure out the system behavior.

Fig. 1 shows the result of applying an ac current to the sample with one vortex per unit cell. The ac current is applied in the $\mathrm{y}$-axis direction (perpendicular to the triangle base); hence the Lorentz force is parallel to the triangle base ( $\mathrm{x}$-axis direction). The vortex motion is in the direction parallel to the triangle base. Apart from sub $-\mu \mathrm{V}$ noise, Fig. 1 shows that there is no rectified voltage (dc voltage) for any value of the ac current amplitude for this orientation, in contrast to the case of the other current orientation shown in Fig. 2. The ratchet effect is not observed in the case of Fig. 1, even though the current is well above the critical current value (see inset of Fig. 1. Inset shows I, V curve and the critical current criterion). The vortex motion is on symmetric potential. But if the ac current is applied along the $\mathrm{x}$-axis direction, the vortex motion takes place in the y-axis direction (see Fig. 2) and a de voltage appears. In this last case, vortex motion is on asymmetric potentials and a ratchet effect occurs. The vortex entrance and exit in the pinning center is strongly asymmetric in this case. The array of $\mathrm{Ni}$ triangles induces a net vortex motion under external fluctuations in the lack of any driving direct forces: injecting an ac current yields a dc voltage.

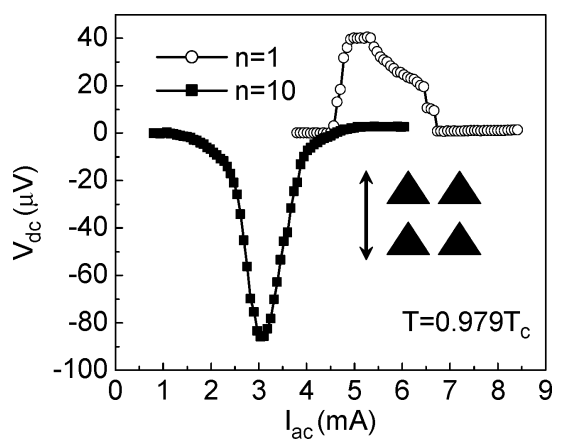

Fig. 2. Rectification effect for the sample shown in Fig. 1 (Critical temperature is $\left.\mathrm{T}_{\mathrm{c}}=8.34 \mathrm{~K}\right)$. The double arrow shows the vortex motion direction. The experimental data correspond to applied fields at the first matching field $(\mathbf{n}=1)$ and $\mathrm{n}=10$ (ten vortices per array unit cell). The magnetic field values are $32 \times 10^{-3} \mathrm{~T}(\mathbf{n}=1)$ and $32 \times 10^{-2} \mathrm{~T}(\mathbf{n}=10)$.

Even more interesting is the effect induced by increasing the applied magnetic field (see Fig. 2). In Fig. 2 we show the effect due to the first matching field ( $\mathrm{n}=1$, one vortex per unit cell) and the effect induced at $n=10$ (10 vortices per unit cell). The dc voltage reverses and the device shows a negative polarity. The rectification polarity could be tuned by the applied magnetic field. This effect is due to the interplay between vortices placed in the pinning centers and interstitial vortices [1]. That is, these $\mathrm{Ni}$ triangles could accommodate a maximum number of vortices (three for this triangle dimension) and the vortex excess is located among triangles as interstitial vortices. The interstitial ones feel a weaker and a reversed asymmetric pinning potential, therefore they move easier and first in comparison with the pinned ones. The depinning applied current (related with the Lorentz force) is smaller for interstitial vortices than for pinned ones to get a de signal and this is exactly the result shown in Fig. 2. This device works as a reversible rectifier. The polarity of the output voltage can be changed by the strength of the ac bias current and applied magnetic field as long as interstitial vortices and pinned ones coexist.

Once the background behavior has been established we can focus on other key questions. Among them, we will address the frequency dependence, the influence of the magnetic character of the pinning center, the temperature window and the applied ac current window in which the effect is revealed.

In our experimental set up, the highest available frequency is $\mathrm{f}=10 \mathrm{kHz}$ and the effect does not show any experimental change below this frequency. Even more, we can study the effect in the so-called adiabatic regime $(f \rightarrow 0)$. In Fig. 3 this experimental behavior is shown. We have checked the ratchet potential injecting the dc current in both senses (I+) and (I-), that is, applying the dc current in the $+\mathrm{x}$-axis and $-\mathrm{x}$-axis direction respectively. Fig. 3(b) shows the dc V(I+) and the dc V(I-) experimental curves. In Fig. 3(a) both curves are subtracted and the net voltage $V=V(I+)-|V(I-)|$ is extracted, so we are dealing with the so-called adiabatic ratchet. The ac and dc signal mimic each other. The unique expected frequency effect would occur at very high frequency, when the period of the ac current becomes similar to the separation between pinning centers. Another conclusion, from the comparison between the y-axis scale in Fig. 3(a) and Fig. 3(b), is that the ratchet signal is one or two order of magnitude lower than the background signal. 


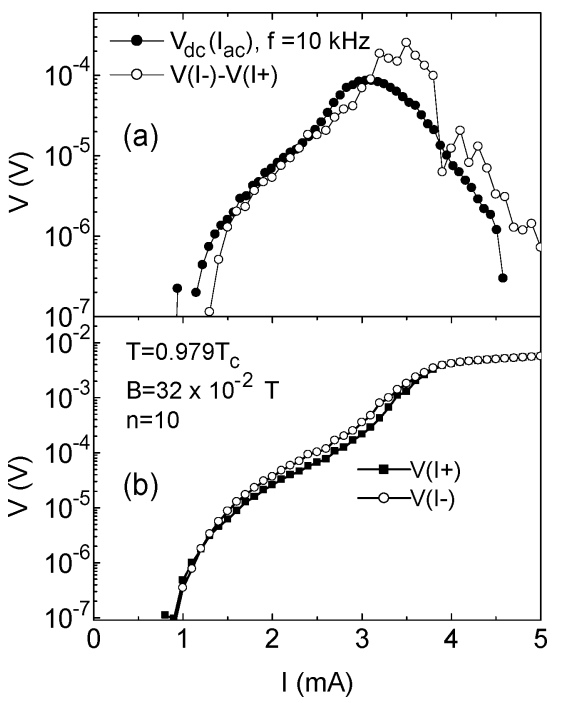

Fig. 3. Data obtained for the sample shown in Fig. 1 (Critical temperature is $\mathrm{T}_{\mathrm{c}}=8.34 \mathrm{~K}$ ). (a) Comparison between the (ac) ratchet effect $(\bullet)$ and the (dc) adiabatic limit (o). (b) Experimental V(I) curves for opposite senses of the dc driving current.

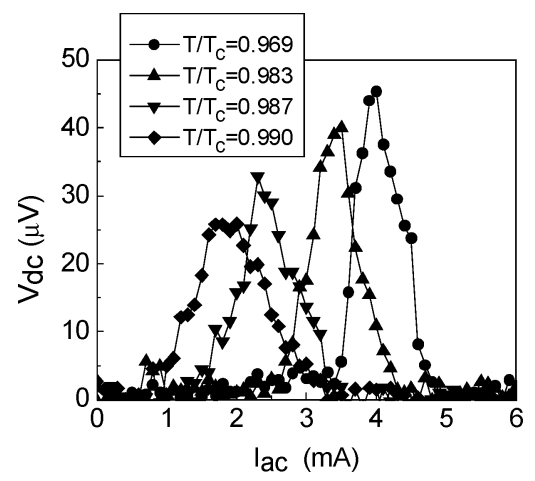

Fig. 4. Data obtained for a $\mathrm{Nb}$ film deposited on an array of $\mathrm{Cu}$ triangles $(660$ $\mathrm{nm}$ side size) with periods of $833 \mathrm{~nm} \times 730 \mathrm{~nm}$. The critical temperature of the sample is $T_{c}=8.25 \mathrm{~K}$. The graph shows the ac current amplitude and temperature dependence of the rectification effect.

This rectifier could be a very promising device depending on the temperature range over the ratchet effect works and whether or not the magnetic pinning centers could be replaced by nonmagnetic pinning potentials without losing efficiency.

To test these points we have fabricated $\mathrm{Nb}$ films on similar array of triangle pinning potentials, but the used material was nonmagnetic $\mathrm{Cu}$ instead of magnetic $\mathrm{Ni}$. The data shown in Fig. 4 correspond to an array of $\mathrm{Cu}$ triangles at the first matching field. The first remarkable fact is that the ratchet effect can be observed too when the asymmetric pinning potential is generated by nonferromagnetic pinning centers. Magnetic materials in superconducting devices could enhance the noise in these devices; therefore to improve them a clear requirement is to eliminate magnetic materials from these devices.

Finally, the experimental current and temperature windows could be studied from Fig. 4 data. About these points, we want to stress that the same trends are observed in the case of samples grown on magnetic pinning centers.
Decreasing the temperature, the rectification effect is enhanced and the maximum driving force is shifted to higher values. This behavior limits the temperatures and driving current strengths where the device works. Vortex flow in samples with artificially induced periodic pinning potentials is a well-known topic. In these samples the vortex dynamics is governed by the interplay among different pinning mechanisms and temperature [13]-[15]. The competition among the random intrinsic pinning, the periodic artificially induced pinning mechanisms and the temperature plays the crucial role in order to establish the experimental window limits. The vortex motion rectification always happens close to the superconducting critical temperature where the periodic potentials can overcome the intrinsic random pinning mechanism, that is, close to the low critical current region, where the vortex lattice can be depinned easily. Very close to the critical temperature, the temperature effect dominates and the ratchet effect is washed out. Then, the high temperature reduces the magnitude of the ratchet effect and decreasing the temperature the effect is increased. On the other hand, the depinning force is enhanced decreasing the temperature; therefore the driving force (driving current) is shifted to higher values to overcome this stronger pinning effect.

In summary, an ac-dc reversible rectifier has been fabricated using $\mathrm{Nb}$ film grown on magnetic and nonmagnetic array of asymmetric pinning potentials. This device is based on the adiabatic ratchet effect and works close to the superconducting critical temperature. This system could be a good candidate to different kind of electronic superconducting devices based on the vortex motion control.

\section{REFERENCES}

[1] J. E. Villegas, S. Savel'ev, F. Nori, E. M. Gonzalez, J. V. Anguita, R. Garcia, and J. L. Vicent, "A superconducting reversible rectifier that controls the motion of magnetic flux quanta," Science, vol. 302, pp. 1188-1191, 2003.

[2] S. Matthias and F. Müller, "Asymmetric pores in a silicon membrane acting as massively parallel Brownian ratchets," Nature, vol. 424, pp. 53-57, 2003.

[3] H. Linke, T. E. Humphrey, A. Löfgren, A. O. Sushkov, R. Newbury, R. P. Taylor, and P. Omling, "Experimental tunneling ratchets," Science, vol. 286, pp. 2314-2317, 1999.

[4] C. S. Lee, B. Janko, L. Derényi, and A. L. Barabasi, "Reducing vortex density in superconductors using the ratchet effect," Nature, vol. 400, pp. 337-340, 1999.

[5] F. J. Wambaugh, C. Reichhardt, C. J. Olson, F. Marchesoni, and F. Nori, "Superconducting fluxon pumps and lenses," Phys. Rev. Lett., vol. 83, pp. 5106-5110, 1999.

[6] B. Y. Zhu, F. Marchesoni, and F. Nori, "Controlling the motion of magnetic flux quanta," Phys. Rev. Lett., vol. 92, no. 1-4, p. 180602 , 2004.

[7] J. S. Weiss, D. Koelle, J. Müller, R. Gross, and K. Barthal, "Ratchet effect in dc SQUIDs," Europhys. Lett., vol. 51, pp. 499-505, 2000.

[8] G. Carapella and G. Costabile, "Ratchet effect: demonstration of a relativistic fluxon diode," Phys. Rev. Lett., vol. 87, pp. 5106-5110, 2001.

[9] F. Falo, P. J. Martinez, J. J. Mazo, T. P. Orlando, K. Segall, and E. Trias, "Fluxon ratchet potentials in superconducting circuits," Appl. Phys. A, vol. 75, pp. 263-270, 2002.

[10] R. Wordenweber, P. Dymashevski, and V. R. Misko, "Guidance of vortices and the vortex ratchet effect in high $T_{C}$ superconducting films obtained by arrangement of antidots," Phys. Rev. B, vol. 69, no. 1-6, p. $184504,2004$. 
[11] J. I. Martin, J. Nogues, K. Liu, J. L. Vicent, and I. K. Schuller, “Ordered magnetic nanostructures: fabrication and properties," J. Magn. Magn. Mater, vol. 256, pp. 449-501, 2003.

[12] J. I. Martin, M. Velez, J. Nogues, and I. K. Schuller, "Flux pinning in a superconductor by an array of submicrometer magnetic dots," Phys. Rev. Lett., vol. 79, pp. 1929-1932, 1997.

[13] J. I. Martin, M. Velez, A. Hoffmann, I. K. Schuller, and J. L. Vicent, "Artificially induced reconfiguration of the vortex lattice by arrays of magnetic dots," Phys. Rev. Lett., vol. 83, pp. 1022-1026, 1999.
[14] Y. Jaccard, J. I. Martin, M. C. Cyrille, M. Velez, J. L. Vicent, and I. K. Schuller, "Magnetic pinning of the vortex lattice by arrays of submicrometric dots," Phys. Rev. B, vol. 58, pp. 8232-8235, 1998.

[15] J. I. Martin, M. Velez, A. Hoffmann, I. K. Schuller, and J. L. Vicent, "Temperature dependence and mechanisms of vortex pinning by periodic arrays of Ni dots in Nb films," Phys. Rev. B, vol. 65, pp. 9110-9116, 2000.

[16] M. Vélez, D. Jaque, J. I. Martín, F. Guinea, and J. L. Vicent, "Order in driven vortex lattices in superconducting $\mathrm{Nb}$ films with nanostructured pinning potentials," Phys. Rev. B, vol. 65, no. 1-5, p. 094 509, 2002. 